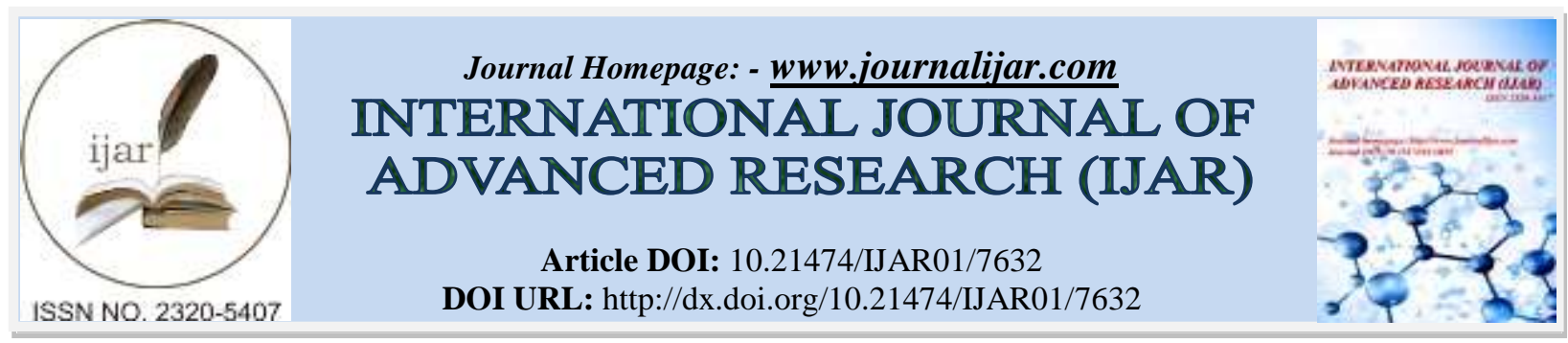

RESEARCH ARTICLE

\title{
TUMEUR DE BUSCHKE LOWENSTEIN: A PROPOS DE DEUX CAS.
}

\author{
Chimae Eddaoudi ${ }^{1}$, Jaouad Lafquir ${ }^{2}$, Abdelhay Filali ${ }^{1}$, Said Benamer ${ }^{2}$, Zaki EL Hanchi ${ }^{2}$ and Hafid Hachi ${ }^{2}$. \\ 1. maternité universitaire des orangers, CHU Ibn Sina, université Mohammed V, Rabat, Maroc. \\ 2. pôle gynéco-mammaire, institut national d'oncologie, Rabat, Maroc.
}

\section{Manuscript Info}

Manuscript History

Received: 24 June 2018

Final Accepted: 26 July 2018

Published: August 2018

Keywords:-

Vulve,Buschke Lownestein,

condylomes, vulvectomie.

\begin{abstract}
La tumeur de Buschke Lownestein (TBL) est un condylome acuminé géant caractérisé par son potentiel dégénératif et son caractère envahissant et récidivant après traitement. C'est une affection rare, de déterminisme viral transmise essentiellement par voie sexuelle. Nous rapportons deux cas de TBL au niveau de la région vulvaire.
\end{abstract}

Copy Right, IJAR, 2018,. All rights reserved.

\section{Introduction:-}

Le condylome acuminé géant ou tumeur de Buschke Lowenstein (TBL) est une entité clinique rare, d'origine virale, transmise essentiellement par voie sexuelle, Une infection par human papillomavirus (HPV) 6 et 11 est volontiers associée à ces tumeurs. Le risque d'envahissement local, le fort pouvoir récidivant et le potentiel dégénératif expliquent sa gravité. Elle se caractérise par la fréquence des récidives et le risque de transformation maligne. Le traitement est essentiellement chirurgical, il est difficile même si l'histologie confirme la bénignité. La chirurgie doit être suffisamment large voire mutilante pour écarter le spectre de récidive et espérer une guérison définitive.

\section{Observation $\mathbf{n}^{\circ}$ 1:}

Patiente âgée de 33ans, célibataire, nulligeste, de bas niveau socio-économique,notion de rapports sexuels non protégés, ayant comme antécédents pathologiques des condylomes vulvaires traités par électrocoagulation, consulte pour réapparition d'une tuméfaction vulvaire, à l'examen présence de tumeur vulvaire bourgeonnante en chou-fleur occupant les grandes lèvres allant jusqu'au mont de pubis (fig1),l'examen au spéculum note la présence d'un ectropion ,le reste de l'examen gynécologique et somatique est sans particularité, la patiente a bénéficié des sérologies VIH et syphilis revenues négatives ,un frottis cervical a été réalisé objectivant un aspect ASC-US, complété par un typage viral qui a détecté 14 types d'HPV à haut risque, la patiente a bénéficié donc d'une colposcopie+biopsie objectivant une lésion CIN 2, une TDM abdomino-pelvienne a été réalisée objectivant un kyste ovarien gauche de densité liquidienne mesurant $40 \mathrm{~mm}$ renfermant une fine cloison, la décision thérapeutique était de réaliser une vulvetomie partielle (fig 2,3) associée à une conisation, le résultat de l'examen anatomopathologique de vulvectomie était en faveur de condylomes, absence de signes de malignité, limites d'exérèse latérale et profonde saines. le résultat de l'examen anatomopathologique de la conisation objectivait une lésion intra-épithéliale de bas grade et focalement de haut grade (CIN 2) étendu aux glandes endocervicales,la décision était de garder la patiente sous surveillance par des frottis cervicaux tous les 3 mois. 


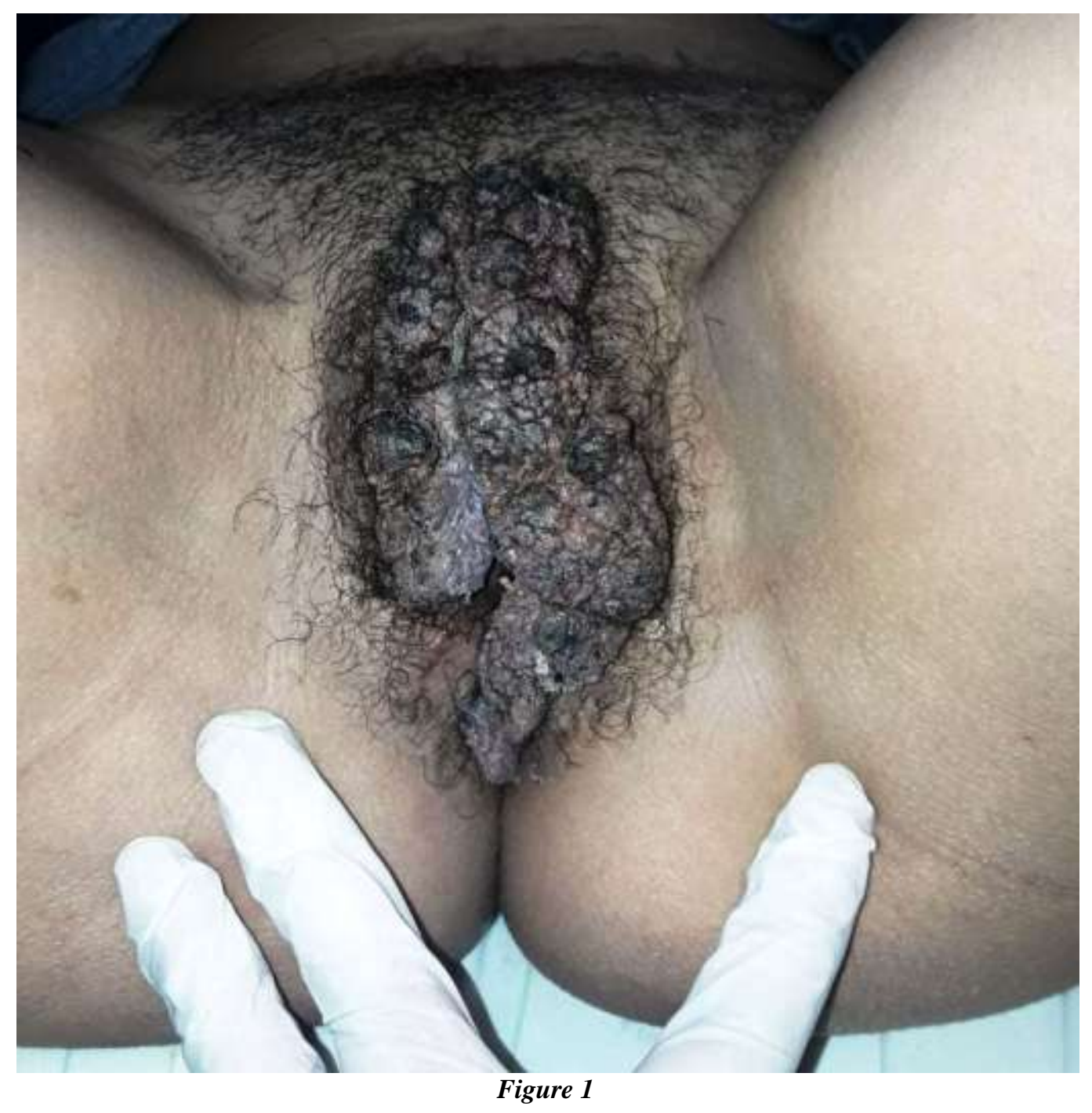




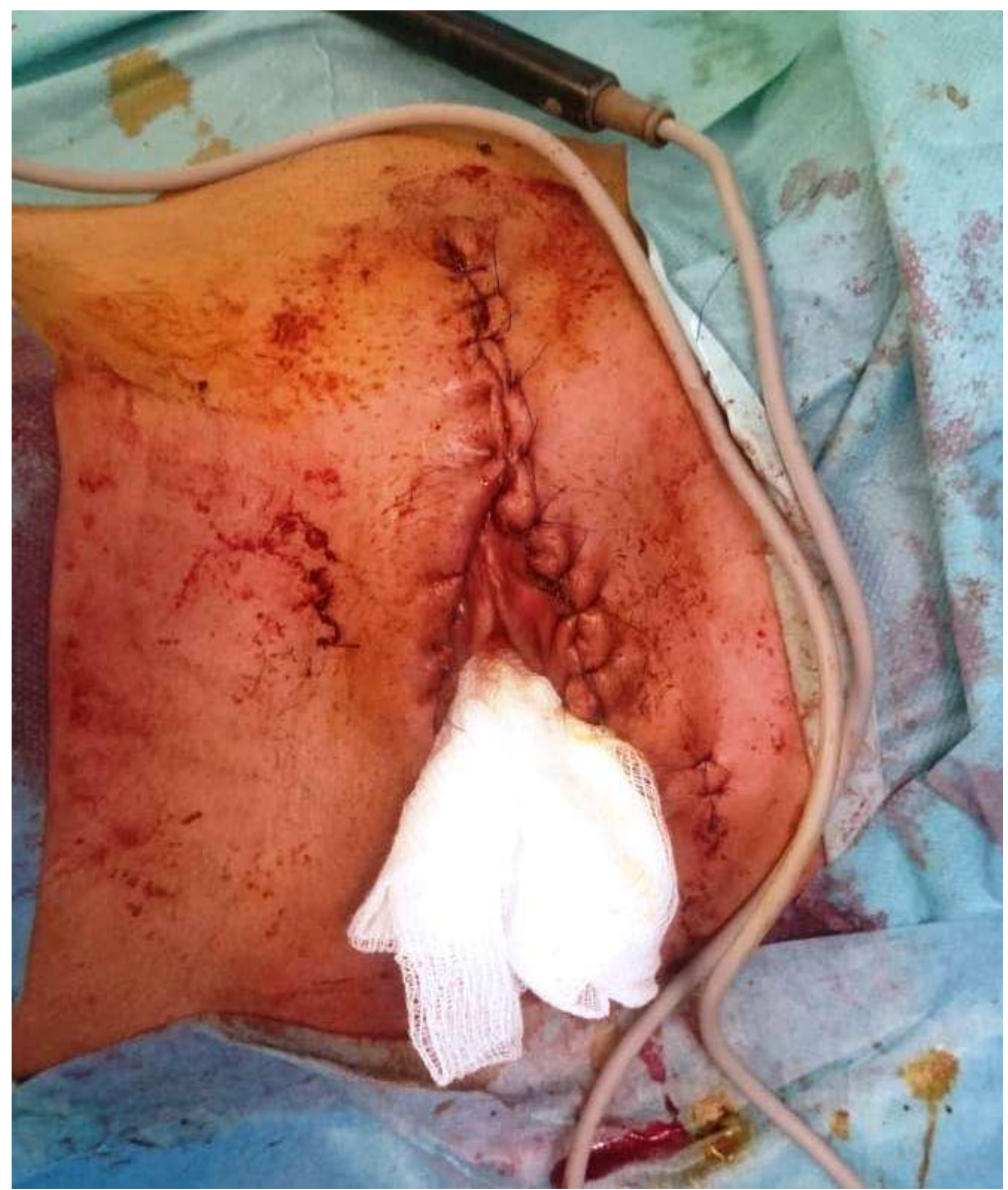

Figure 2 


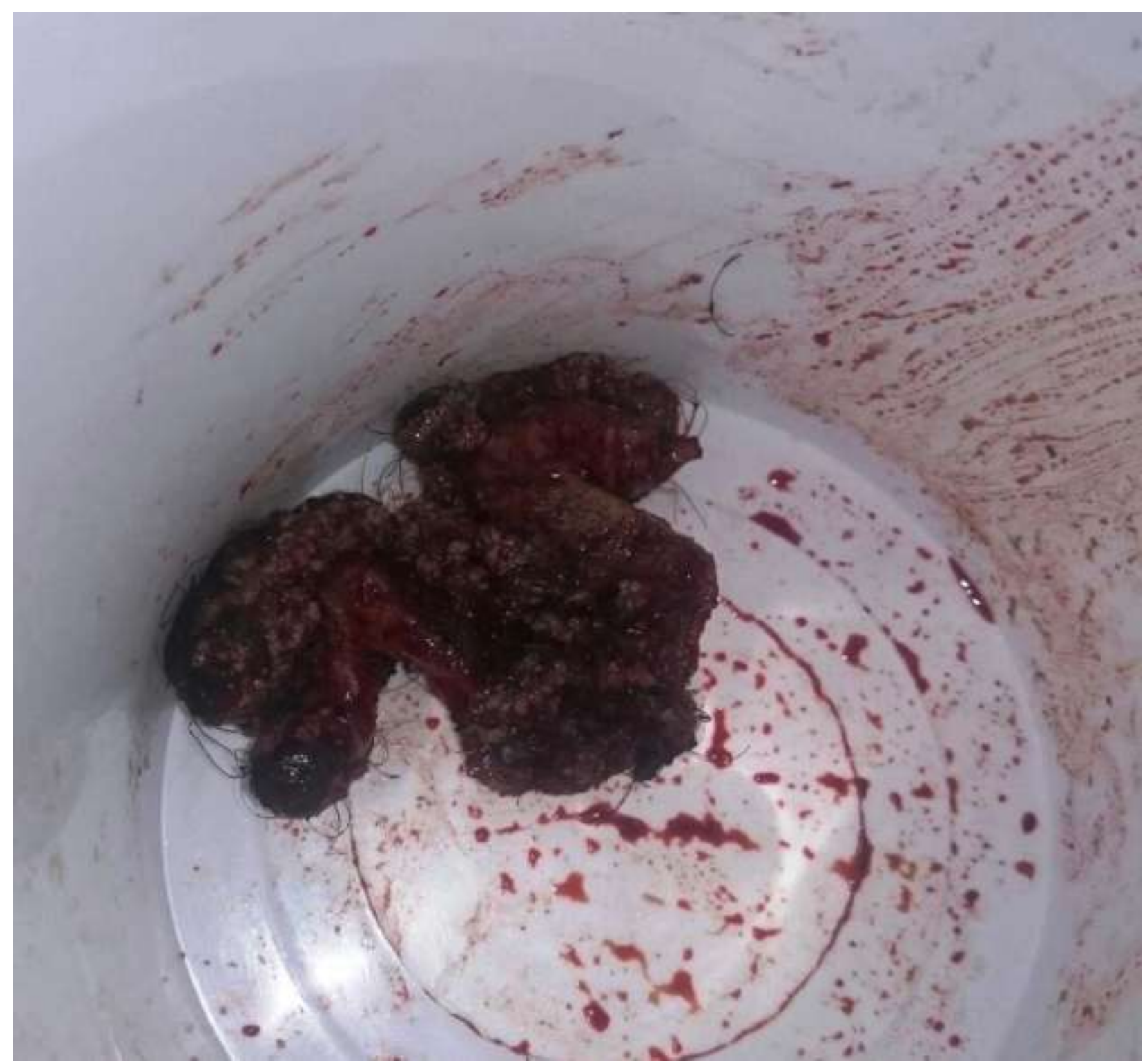

Figure 3

\section{Observation $\mathbf{n}^{\circ} 2$ :}

Patiente âgée de 50ans, mariée,multipare, suivie pour sarcoïdose sous corticoïdes ,pas de notion de comportements sexuels à risque, qui présente depuis un an une lésion vulvaire qui a progressé sur toute la vulve et la marge anale, à l'examen présence de lésions condylomateuses occupant toute la région vulvaire allant jusqu'au l'anus (fig 4), la biopsie a objectivé une TBL, les sérologies VIH ,syphilis et hépatite B et C sont négatives, le frottis trouve un aspect ASC-US, la colposcopie n'objective pas de lésions suspectes, la patiente a bénéficié d'une vulvectomie totale (fig $5,6,7,8)$, le résultat d'examen anatomopathologique trouve une TBL sans signes de transformation maligne, limites d'exérèse latérale et profonde saines. 


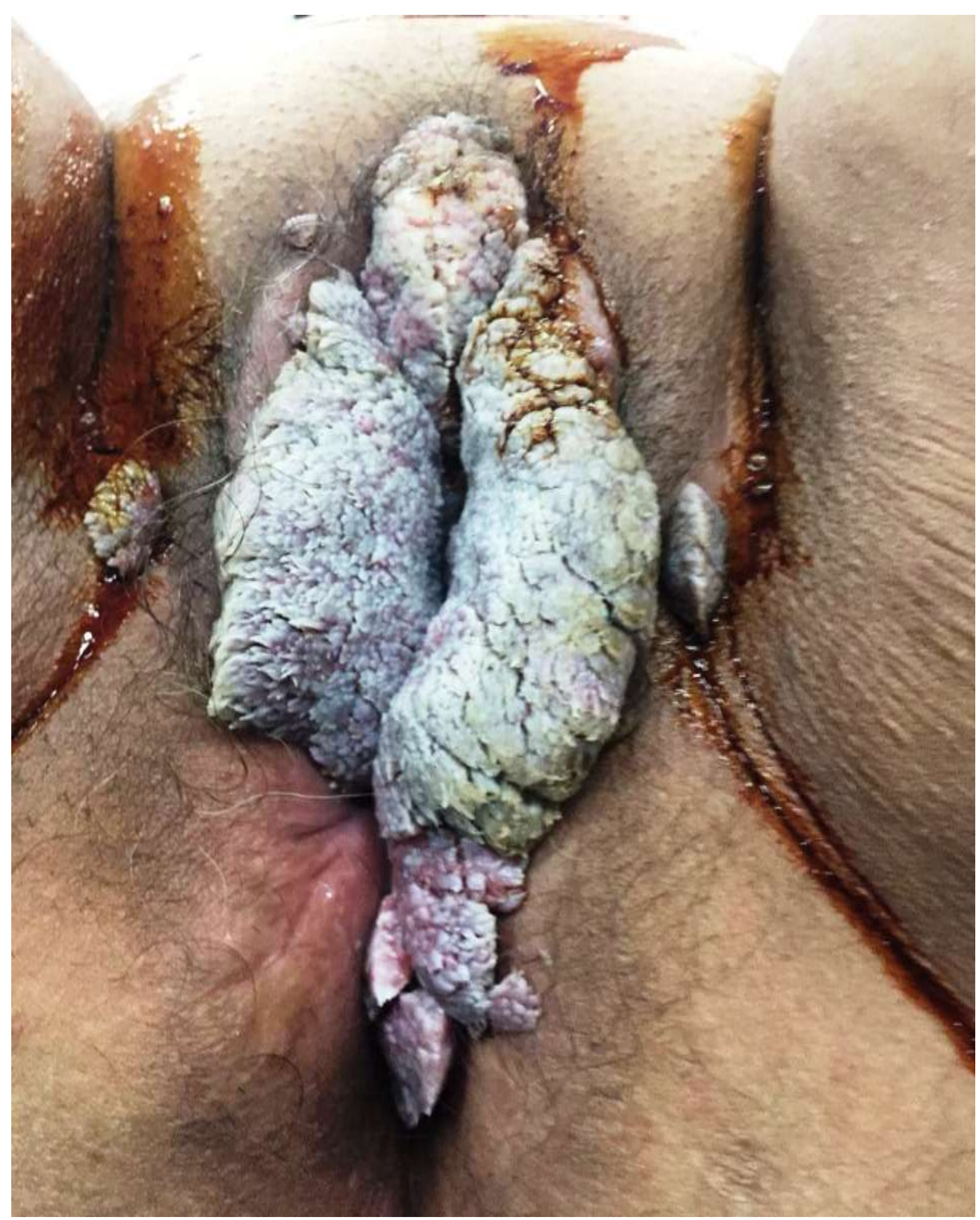

Figure 4 


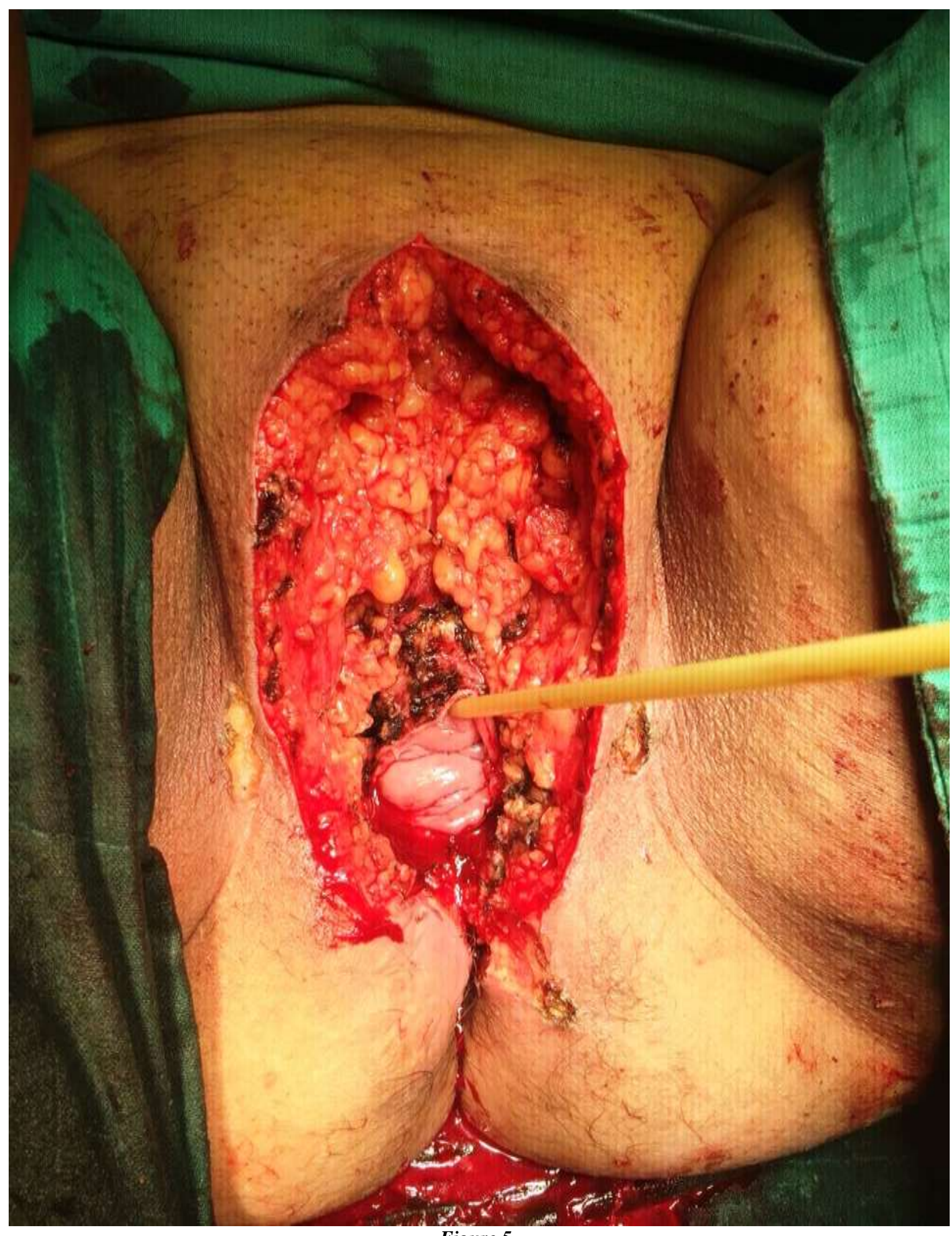

Figure 5 


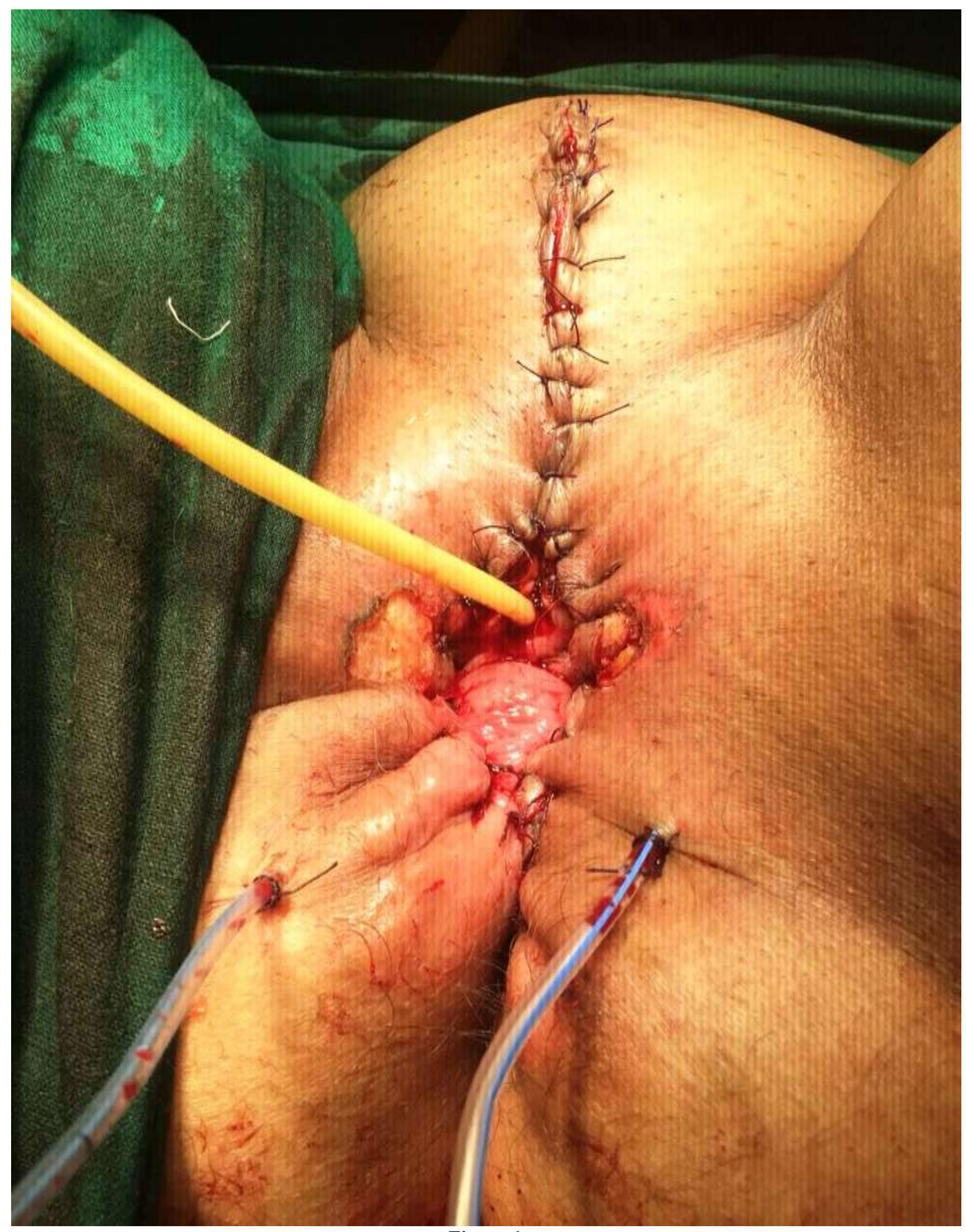

Figure 6 


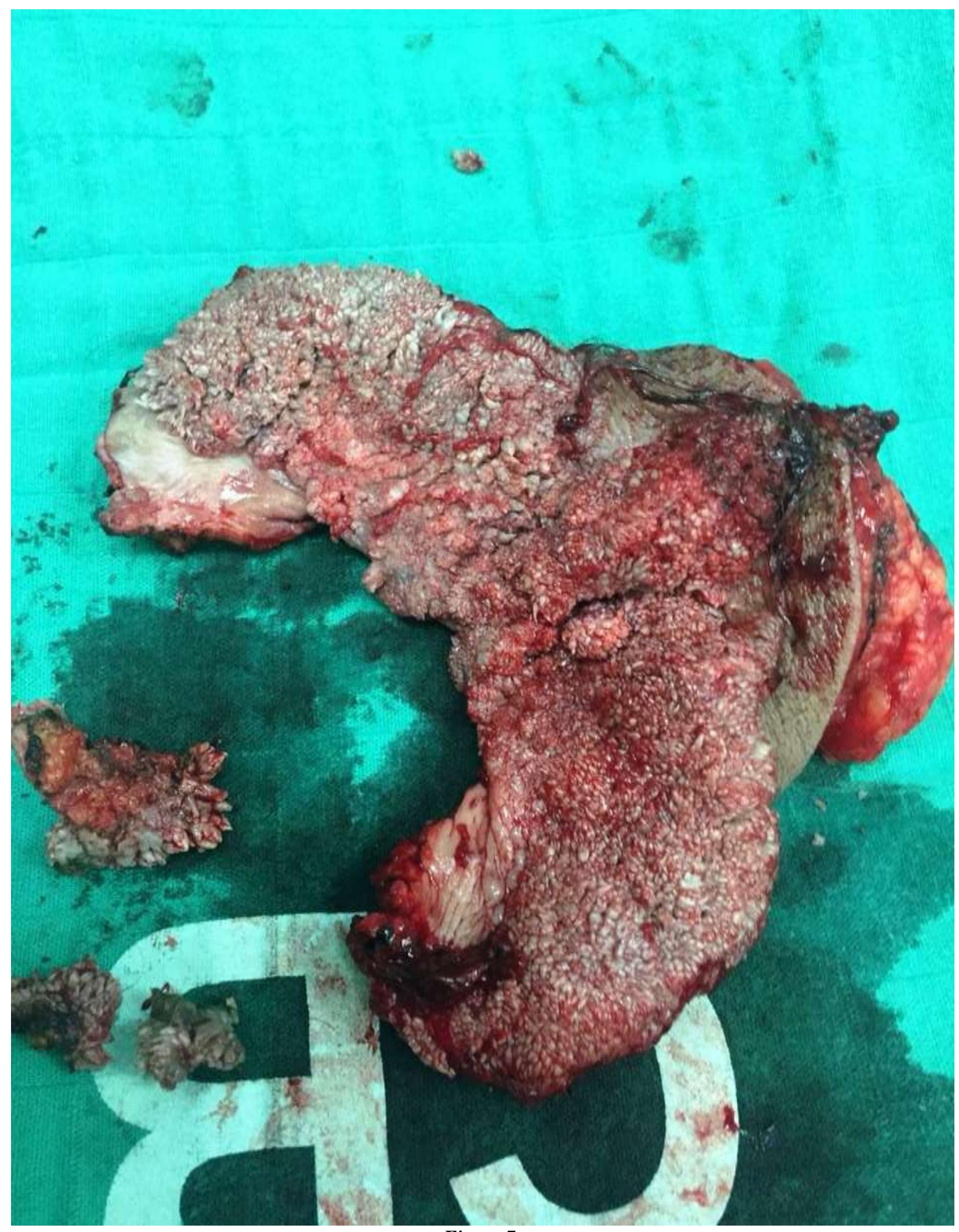

Figure 7 


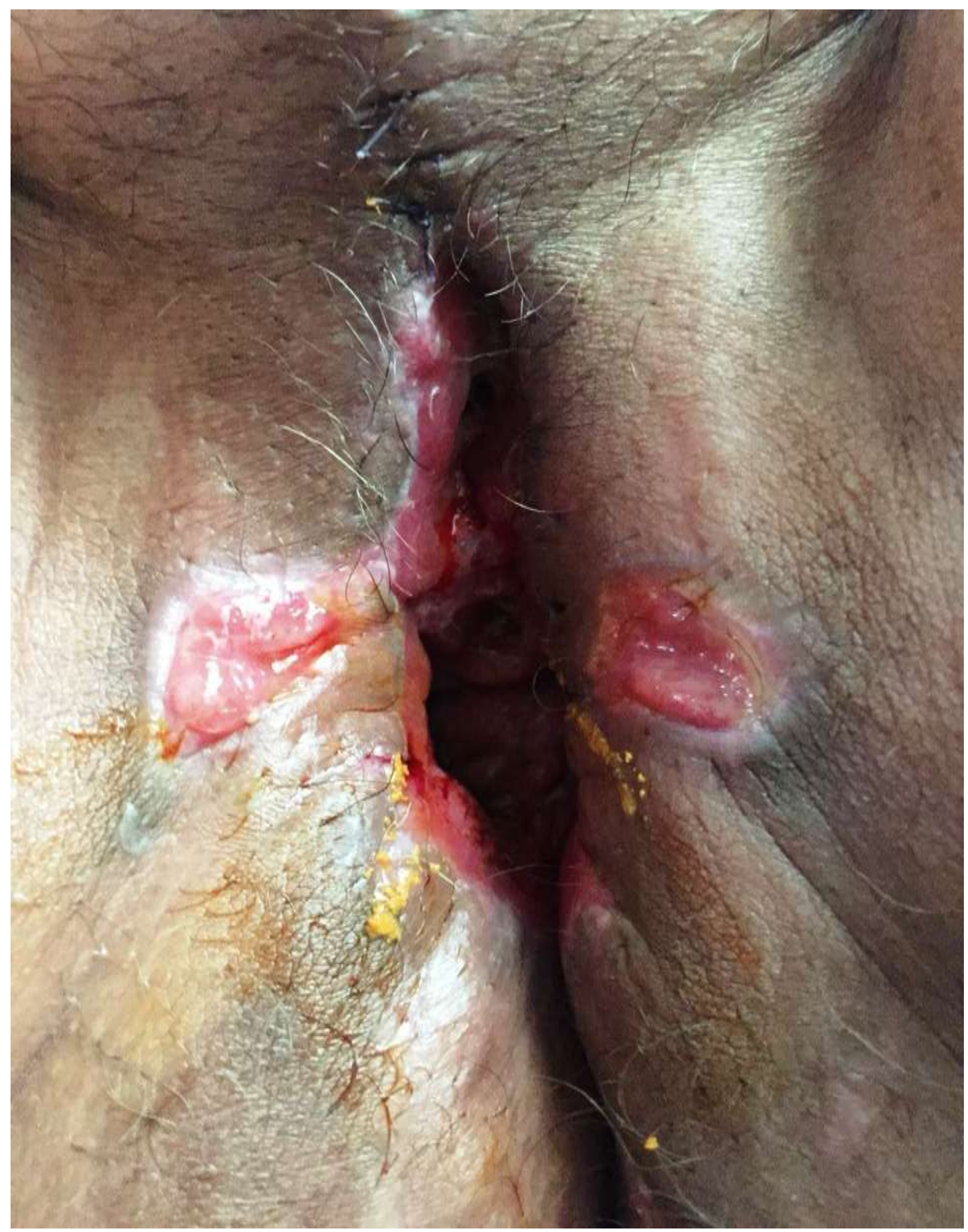

Figure 8:- aspect après cicatrisation 


\section{Discussion:-}

La tumeur de Buschke-Löwenstein a été décrite pour la première fois par Buschke et Lowensteinin en 1925. Ils ont observé une lésion du pénis ressemblant cliniquement à la fois aux condylomes acuminés (verrues vénériennes) et aux carcinomes épidermoïdes, mais avec des caractéristiques biologiques et aspect anatomo-pathologique différents.(1).

Le condylome acuminé géant ou tumeur de Buschke-Löwenstein (TBL) est une entité nosologique rare, sexuellement transmissible. Il s'agit d'une infection virale induite par le papillomavirus humain (HPV) dont les sérotypes les plus fréquents sont le 6,11, 16 et 18.(2).

La tumeur se caractérise cliniquement par une lésion bourgeonnante plus ou moins ulcérée de la région périanale ou génitale. Le sex-ratio hommes-femmes est approximativement de 2,3/1. La tumeur se localise chez l'homme le plus souvent au niveau de la région balanopréputiale, débutant sous forme de condylomes, s'étendant progressivement au gland, au prépuce et au fourreau de la verge. Chez la femme, la localisation la plus fréquente est vulvaire, s'étendant parfois jusqu'à la région périanale.(3).

La tumeur s'étend en surface et en profondeur, et peut se compliquer d'un envahissement des structures de voisinage, de surinfection et de fistulisation aux organes voisins. Une transformation en tumeur maligne a été rapportée dans 30 à $56 \%$ des cas parmi des séries de tumeurs de BuschkeLöwenstein périanales. $(4,5)$ Les récidives locales après exérèse de la lésion initiale sont fréquentes, peuvent atteindre $65 \%$ dans les localisations périanales(4).Les risques de métastase ganglionnaire sont très faibles, les métastases viscérales sont exceptionnelles. Sur le plan histologique la TBL est à l'origine de lésions d'acanthose, de papillomatose, d'hyperkératose parakératosique avec présence de koïlocytes qui sont pathognomoniques de l'infection par HPV, à noyau chiffonné avec un halo clair périnucléaire $(6,7)$.

Le traitement de choix est chirurgical (3). L'exérèse doit être la plus large possible, emportant une marge de tissu sain (3) confirmé par l'examen anatomopathologique.la chirurgie doit être suffisamment large voire mutilante pour écarter le spectre de récidive et espérer une guérison définitive. Elle est variable selon la localisation . Le caractère complet de l'exérèse chirurgicale de la TBL doit être confirmé par l'examen anatomopathologique de la pièce opératoire. Si l'exérèse chirurgicale est incomplète, la reprise chirurgicale est indiquée (8).

Les topiques locaux (podophyline, 5FU), l'électrocoagulation, la cryothérapie, et la destruction au laser largement utilisés dans le traitement des condylomes banaux sont inefficaces dans le traitement de la TBL . Ces moyens ont l'inconvénient majeur de ne pas fournir un tissu pour l'analyse histologique (8)..

La chimiothérapie à base de méthotrexate ou de bléomycine peut être utilisée mais elle reste sans véritable apport, elle est surtout indiquée en préopératoire afin de réduire le volume tumoral et de diminuer l'agressivité de l'acte chirurgical . L'utilisation de la radiothérapie est controversée . Elle est utilisée en préopératoire pour diminuer la masse tumorale, ou en dernier recours pour des tumeurs non opérables. Pour certains auteurs, L'association de la radiothérapie à la chimiothérapie en néoadjuvant pourrait donner des résultats remarquables, elle a été utilisée avec succès pour traiter des TBL dégénérées (8). Pour d'autres, La chimiothérapie et la radiothérapie ne doivent être administré que dans les TBL non résécables ou récidivantes puisque leur efficacité n'a pas été pleinement documenté.

L'immunothérapie par autovaccination semble avoir une certaine efficacité dans le traitement des condylomes anciens et récidivants.

Les récidives sont l'une des caractéristiques de cette tumeur, elles sont la conséquence directe d'un geste chirurgical trop limité . Le risque de récidives après excision est de 60 à $66 \%$ (8).

La fréquence des récidives et le risque de survenue de carcinome épidermoïde imposent une surveillance clinique (locorégionale) et histologique prolongée (6). 


\section{Conclusion:-}

La TBL est une tumeur rare, d'origine virale, à transmission sexuelle, qui peut toucher l'homme et la femme, le traitement est avant tout chirurgical, et doit être large du fait du haut potentiel récidivant de la tumeur, des traitements néoadjuvants à type de chimiothérapie ou immunothérapie mériteraient d'être mieux évalués pour limiter le caractère délabrant de la chirurgie. Le risque de récidive et la transformation potentielle en carcinome spinocellulaire imposent un suivi régulier de ces patients.

\section{Références:-}

1. Loewenstein LW. Carcinoma-like condylomata acuminate of the penis. Med Clin N Am 1939;23:789-95

2. Rimtebaye $\mathrm{K}$, et al. Tumeur de Buschke-Löwenstein: à propos de 8 cas et revue de la littérature. Afr J Urol (2016), http://dx.doi.org/10.1016/j.afju.2016.01.013

3. Lévy, C. Lebbe. Prise en charge des tumeurs de Buschke-Löwenstein. Annales d'urologie - EMC Urologie 40 (2006) $175-178$

4. Creasman C, Haas PA, Fox TA, Balazs M. Malignant transformation of anorectal giant condyloma acuminatum (BuschkeLöwenstein tumor). Dis Colon Rectum 1989;32:481-7

5. Chu QD, Vezeridis MP, Libbey NP, Wanebo HJ. Giant condyloma acuminatum (Buschke-Löwenstein tumor) of the anorectal and perianal regions: analysis of 42 cases. Dis Colon Rectum 1994;37:950-7.

6. El mejjad A, Dakir M, Tahiri M, Attar H. Le condylome acuminé géant-Tumeur de Buschke-Lowenstein (à propos de 3 cas). Prog Urol 2003;13:513-7.

7. Frega a, Stentella P, Tinari A, Vecchione A, Marchionni M. Giant condyloma acuminatum or buschkelowenstein tumor: review of the literature and report of three cases treated by $\mathrm{CO} 2$ laser surgery. A long-term follow-up. Anticancer Res 2002;22:1201-4.

8. Noureddine Njoumi et al. La tumeur de Buschke-Lowenstein anorectale : à propos de 16 cas et revue de la littérature. The Pan African Medical Journal. 2013;16:131. 